\title{
Oromucosal Route of Administration
}

National Cancer Institute

\section{Source}

National Cancer Institute. Oromucosal Route of Administration. NCI Thesaurus. Code C64906.

Administration of a medicinal product to the oral cavity to obtain either a systemic or a local effect. 